\title{
ARCHITETTURA RURALE E VALORIZZAZIONE DEL PATRIMONIO CULTURALE IN SICILIA
}

\author{
PIETRO CALAMIA \\ Università degli Studi di Palermo
}

Abstract

Il contributo si propone di esaminare i risultati che gli interventi di riqualificazione del patrimonio culturale apportano all'architettura rurale e al paesaggio siciliani, focalizzando l'attenzione sulla eventuale capacità di restituire centralità e adeguato valore al territorio e alla terra in quanto risorsa multifunzionale, in grado di assicurare servizi fondamentali per il benessere delle comunità locali, e di conservare il patrimonio storico, culturale e paesaggistico. A tal fine, si analizza in maniera dettagliata un caso studio di riqualificazione di un manufatto architettonico presente sul territorio del paesaggio rurale siciliano.

Parole chiave

Architettura rurale, recupero edilizio, patrimonio storico

\section{ARQUITECTURA RURAL Y PUESTA EN VALOR DEL PATRIMONIO CULTURAL EN SICILIA}

\section{Resumen}

La contribución pretende examinar los resultados que las medidas de puesta en valor del patrimonio cultural aportan a la arquitectura rural y al paisaje sicilianos, centrándose en la mejora de la construcción en las zonas rurales y sobre la posible capacidad de devolver centralidad y valor adecuado al territorio, en quanto recurso multifuncional, capaz de garantizar servicios fundamentales para el bienestar de las comunidades locales y de conservar el patrimonio histórico, cultural y paisajístico. A tal efecto, se analiza un caso de estudio de recalificación de un ejemplo arquitectónico siciliano.

\section{Palabras clave}

Arquitectura rural, recalificación de edificios, patrimonio histórico.

\section{RURAL ARCHITECTURE AND ENHANCEMENT OF CULTURAL HERITAGE IN SICILY}

\begin{abstract}
The work proposes to examine the results that measures to redevelopment of the cultural heritage brought to the rural architecture and the Sicilian landscape. Attention is focused on the landscape retraining and the possible ability to return centrality and appropriate value to the earth, understood as a multifunctional resource, able to assure essential services for the comfort of the local communities and to preserve the historical, cultural and landscape patrimony. For this purpose, a case study on the subject of the renovation of a Sicilian architectural building is analysed.
\end{abstract}

\section{Keywords}

Rural architecture, building recovery, historical heritage. 


\section{Inquadramento}

Il presente contributo è redatto al fine di fornire alcuni risultati ed i possibili sviluppi degli studi condotti dal sottoscritto, aventi come tema l'architettura, lo sviluppo urbanistico e le opere pubbliche del paesaggio rurale in Sicilia.

L'attualità delle problematiche sui beni culturali ed ambientali e la grande rilevanza che essi rivestono nella Regione Siciliana giustificano e richiedono un grande impegno nella definizione ed attuazione di una politica organica di conservazione e valorizzazione. È infatti indiscutibile l'incidenza che la disciplina dei beni culturali ha sulle "politiche di sviluppo" della Regione Sicilia e, negli ultimi anni, gli interventi di programmazione di derivazione europea stanno assumendo fondamentale importanza nell'accompagnare le politiche di sviluppo del territorio, al fine di rendere più concreti gli interventi di conservazione e valorizzazione.

Nel presente contributo, inizialmente viene svolta un'analisi dello stato dell'arte, con riferimento al territorio e all'architettura rurale; quindi vengono analizzate i contributi che le PC hanno in campo di sviluppo rurale in Sicilia, grazie agli strumenti e alle risorse forniti dalla Comunità Europea. Quindi, si passa all'analisi di un caso studio, mediante il rilievo dello stato di fatto di un fabbricato rurale destinato ad agriturismo e degli interventi a cui è stato sottoposto attraverso gli strumenti e le risorse forniti da bandi legati a finanziamenti della Comunità Europea. Il contributo si propone, in tal modo, di mostrare, proprio attraverso un caso studio, quali siano i risultati apportati al territorio rurale siciliano dai finanziamenti comunitari in tema di sviluppo rurale, focalizzando l'attenzione sulla eventuale capacità di restituire centralità e adeguato valore a tale territorio.

\section{Il patrimonio artistico e culturale del territorio rurale}

La popolazione residente in aree urbane è cresciuta in maniera repentina dalla seconda rivoluzione industriale ad oggi, con andamenti e tempi differenti nei paesi sviluppati o in quelli in via di sviluppo. Gli studi scientifici e le politiche europee, nazionali e regionali hanno mostrato nell'ultimo ventennio un crescente interesse all'analisi dell'efficienza dei modelli insediativi e all'andamento del consumo di suolo, con approcci che considerino molteplici discipline (urbanistica, agronomia, pianificazione territoriale, ecologia del paesaggio, ecc.) ${ }^{1}$. Ogni ambiente antropizzato porta con sé i segni delle attività e del vissuto delle comunità. Pertanto, l'intervento dell'uomo è evidente nella città, ma è ugualmente marcato in quelli che vengono definiti spazi periferici e marginali, quali le campagne.".

Le dinamiche evolutive interessanti il territorio e che si realizzano nell'aumento della componente urbana e antropica, sono generalmente chiamate "urbanizzazione". Del termine "urbanizzazione" si possono trovare diverse definizioni; a livello accademico, Antrop identifica tale fenomeno come un "processo culturale e sociologico che trasforma i paesaggi rurali e naturali in paesaggi urbanizzati" con una conseguente crescita di una nuova tipologia di aree edificate, denominate frange ${ }^{3}$; tale definizione è stata successivamente ripresa e reinterpretata anche da altri autori ${ }^{4}$. Sempre Antrop descrive tale processo assimilandolo ad un ciclo ed individuando alcune fasi che si susseguono nella dinamica evolutiva della città:

- urbanizzazione: concentrazione della popolazione nel centro a seguito della migrazione dalla frangia urbana;

- suburbanizzazione: crescita della popolazione in tutto il centro urbano. Tale crescita non è di tipo uniforme in quanto si registra una perdita di abitanti da parte della zona centrale dell'insediamento e, al contrario, una rapida crescita della popolazione nelle frange urbane;

- disurbanizzazione: iniziale declino complessivo della popolazione urbana, con perdita di abitanti sia nel centro che nelle zone di frangia urbana;

\footnotetext{
${ }^{1}$ Zeng/Sui/Li, 2005: 410-434.

2 Cedrini, 1997: 13.

3 Antrop, 2000: 257-270. Si veda anche Antrop/Van Eetvelde, 2000: 43-58

${ }^{4}$ Slemp et al., 2012: 139-148.
} 
- riurbanizzazione: crescita della popolazione a partire dal centro urbano, seguita da una crescita nella frangia urbana ${ }^{5}$ (fig. 1).

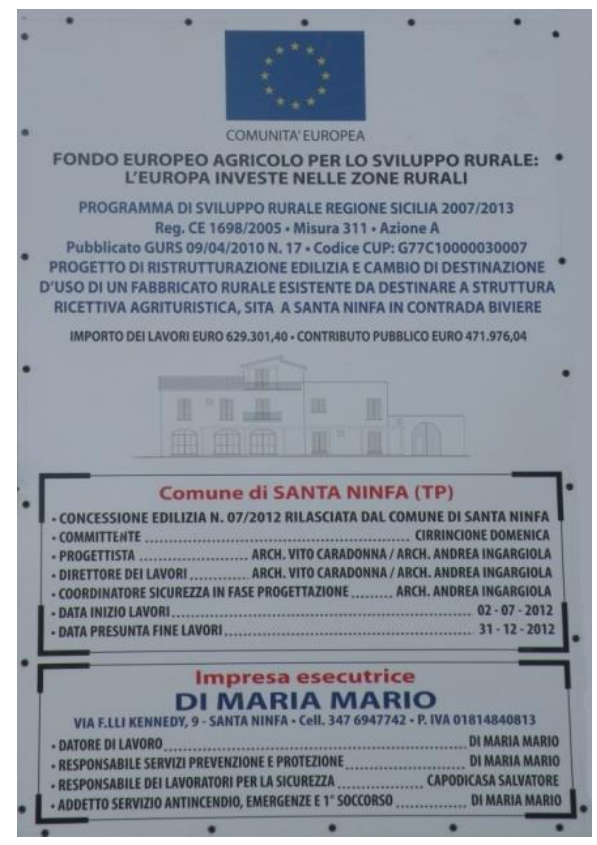

Fig. 1. Foto del cartello informativo di cantiere, Rubino Domenico

L'Unione Europea non ha adottato una classificazione delle unità amministrative sulla base del loro carattere urbano o rurale, ma ha effettuato una suddivisione e codifica gerarchica del territorio degli Stati Membri (prevista dal Reg. CE n.1509/2003) chiamata NUTS (National Territorial Units for Statistic), basata sulla popolazione residente e la gestione amministrativa del territorio stesso.

L'introduzione della categoria di "paesaggio culturale" nei beni patrimoniali dell'Unesco sancisce di fatto una nuova attenzione sociale al paesaggio, anche valorizzando alcune attività locali che possono garantire il rilancio del territorio ${ }^{6}$, superando la contrapposizione manichea che organizzava i beni nelle due grandi categorie dei beni culturali e dei beni naturali, a favore Il paesaggio condensa dentro sé non solo la memoria sociale sedimentata, che è la rappresentazione materiale, visibile e sensibile, della modalità insediativa delle società passate, ma anche l'insieme delle potenzialità di sfruttamento di quel terreno comune ai fini di una convivenza sociale, attraverso la rappresentazione di pratiche condivise che sedimentano o meno prodotti materiali. Tutto ciò entra a far parte del paesaggio culturale da valorizzare e tramandare ${ }^{7}$.

In quest'ottica, l'architettura rurale assume rilevanza in quanto esprime $\mathrm{i}$ segni di un processo storico legato a mutamenti sociali, processi produttivi, relazioni antropologiche che l'uomo instaura con l'ambiente agrario, di cui l'architettura rurale contiene valori e tradizioni. In ragione di ciò, ogni elemento che riguarda l'edificazione e l'espansione di un baglio, di una torre, una casa rurale o di una masseria, è da tenere in considerazione perché fa parte di un processo logico legato all'antropizzazione del territorio. In Sicilia, la città costituisce il polo di attrazione principale attorno al quale ruota la vita economica e sociale delle comunità, lasciando il territorio agricolo (agro) ad uso e consumo dei pochi addetti ai lavori. La città e la campagna appaiono come due lontane entità territoriali, la cui separazione è spesso

\footnotetext{
${ }^{5}$ Antrop, 2004: 9-26.

${ }^{6}$ Berger/Chevalier/Cortes, 2010: 7-16.

${ }^{7}$ Revenga, 2002: 119-123.
} 
accentuata dall'articolazione delle direttrici viarie che si frappongono tra città e campagna, superate le quali inizia uno scenario ambientale fatto di immensi campi di vitigni, uliveti, campi di grano e di altre colture, all'interno dei quali l'architettura dei bagli e delle torri si dispone in modo uniforme. Percorrendo le ex regie trazzere o le mulattiere, osservando gli argini dei due fiumi o l'area delle riserve presenti nel territorio siciliano, si scoprono i segni dell'uomo lasciati nella storia, rappresentati dai tanti siti archeologici, dalle torri, dai bagli. Questi ultimi in particolare appaiano numerosi a chi percorre le strade della campagna siciliana e, anche se molti in precario stato di conservazione, esercitano un particolare fascino, perché conservano una storia fatta da uomini e donne che lì hanno lavorato, hanno faticato, hanno vissuto.

La normativa comunitaria fornisce un importante contributo in tema di conservazione e valorizzazione dei beni culturali. E' evidente come, fin dalle sue origini, l'azione della Comunità intendesse incoraggiare la cooperazione tra gli Stati membri nel settore della conservazione e salvaguardia del patrimonio culturale e artistico di importanza europea, rappresentando un primo passo verso l'idea della necessità di approntare una tutela congiunta dei vari Stati europei su "beni" la cui rilevanza travalichi quella nazionale.

In epoca successiva si comincia ad avvertire anche un secondo tipo di consapevolezza determinato dalla considerazione, sempre più condivisa, che la degradazione o la sparizione di un bene del patrimonio culturale, artistico e naturale di un Paese sia un impoverimento nefasto del patrimonio di tutti i popoli del mondo, e che spesso la protezione di questo patrimonio su scala nazionale rimanga incompleta per l'ampiezza dei mezzi necessari a tal fine e per l'insufficienza delle risorse economiche, scientifiche e tecniche del Paese sul cui territorio il bene da tutelare si trova. Per questo, si mostra indispensabile adottare nuove disposizioni, per lo più di carattere convenzionale, per attuare un efficace sistema di protezione collettiva del patrimonio culturale di valore universale.

Gli obiettivi dell'azione di sostegno dell'Unione Europea sono: innanzitutto promuovere lo sviluppo e l'adeguamento strutturale delle regioni che presentano ritardi; favorire la riconversione economica e sociale delle zone con difficoltà strutturali; favorire l'adeguamento e l'ammodernamento delle politiche e dei sistemi di istruzione, formazione e occupazione.

È indiscutibile l'incidenza che la disciplina dei beni culturali ha sulle "politiche di sviluppo" della Regione Siciliana. Negli ultimi anni le politiche di sviluppo del territorio sono state accompagnate, al fine di rendere più concreti gli interventi di conservazione e valorizzazione, da una programmazione di derivazione europea

Dalla recente direttiva in materia, emergono precise indicazioni in direzione della riconoscibilità degli interventi, della individuazione di tempi e modalità di attuazione, di controlli dei risultati, ecc. In sostanza vengono tracciate linee per eseguire precise e rigorose politiche di programmazione che presuppongono e comportano un'adeguata risposta a livello regionale, attraverso la predisposizione di una trama organizzativa affidabile e funzionale. Si fa quindi riferimento ad un superamento della visione statica degli interventi, attraverso la predisposizione di strategie tendenti alla valorizzazione dei beni ed azioni coordinate allo sviluppo.

In relazione alla tutela e alla valorizzazione dei beni culturali e artitici, è da affrontare anche l'argomento relativo al costruito rurale, riconducibile a "testimonianza avente valore di civiltà'. Infatti dagli anni '60, grazie alla Carta di Venezia e all'estensione della nozione di monumento anche alle "opere modeste che, con il tempo, abbiano acquistato un significato culturale" ", i fabbricati rurali sono entrati a pieno titolo nel novero delle costruzioni da tutelare. Tali strutture architettoniche hanno un valore documentale e artistico, nonché storico culturale, in quanto testimonianza di un mondo, e di un modo di vivere il territorio che si è andato mutando negli ultimi decenni. Inoltre anche la consistenza materiale e tecnologica dell'architettura rurale tradizionale va annoverata quale aspetto di carattere

${ }^{8}$ Carta di Venezia, 1964. 
storico, infatti la povertà e la semplicità dei loro costruttori ha fatto si che fossero adoperati materiali presenti nel luogo o comunque facilmente reperibili, comportando tale circostanza una differenziazione tra regione e regione e una conseguente insostituibile specificità. Anche la loro distribuzione geografica diviene testimonianza di una certa organizzazione sociale, ne è esempio il modello della masseria espressione di una forma di insediamento agricolo dove grande peso assumeva il controllo del territorio da parte dei tenutari delle aziende.

La problematica relativa agli edifici rurali va affrontata tenendo conto delle tipicità di siffatte costruzioni connesse ad un determinato periodo storico e ad una loro peculiare funzione: quella produttiva. Il loro deterioramento è quindi da ricondursi all'inurbamento che ha caratterizzato i tempi moderni e all'industrializzazione dei mezzi produttivi, che ha comportato sempre più un loro progressivo abbandono. Quindi l'esigenza che viene a crearsi, al fine di tutelare tali strutture architettoniche, patrimonio storico dell'isola, è quella della riconversione di tali complessi per una loro diversa più moderna utilizzazione. Da forme di residenza privata a possibilità di sfruttamento per soggiorno turistico, a strutture di uso sociale quali case d'accoglienza per ragazzi a rischio, ma anche strutture per anziani o per attività ludiche infantili e molto altro.

Tra le varie proposte di ristrutturazione di tali edifici la più conforme alle peculiari caratteristiche architettoniche è senza dubbio quella dell'agriturismo, soprattutto per la maggiore continuità con le funzioni originarie. La destinazione agrituristica, quindi, costituisce un'opportunità favorevole per il recupero degli edifici rurali, o di loro parti, in precedenza inutilizzati e quindi destinate all'abbandono. Inoltre prevedendo una varietà di funzioni che vanno dall'alloggio alla ristorazione permette un utilizzo di tutti gli ambienti delle varie strutture rurali, sottolineandone e sfruttandone le caratteristiche tipiche. Per quanto riguarda l'agriturismo quest'ultimo è considerato come una vera e propria attività agricola, accessoria alla coltivazione o all'allevamento, disciplinata dalla L. 5 dicembre 1985 n. 730 (riformata dalla L. n. 268 del 1999 sulla disciplina delle "strade del vino"), dalla legge n.413 del 30/12/1991 (con la quale é stato riconosciuto un regime fiscale apposito) e dalle varie legislazioni regionali che ne discendono.

La normativa definisce come agrituristica ogni attività di "ricezione ed ospitalità esercitate dagli imprenditori agricoli attraverso l'utilizzazione della propria azienda, in rapporto di connessione e complementarità, rispetto alle attività di coltivazione del fondo, silvicoltura, allevamento del bestiame, che devono comunque rimanere principali". Tale normativa pone il miglior uso del patrimonio rurale naturale ed edilizio tra le proprie finalità, rimandando alle leggi regionali la disciplina degli interventi edilizi, precisando che "il restauro deve essere eseguito nel rispetto delle caratteristiche tipologiche ed architettoniche degli edifici esistenti e nel rispetto delle caratteristiche ambientali delle zone interessate".

\section{L'architettura rurale}

Per definire il concetto di architettura rurale non è sufficiente soltanto il richiamo alle attività agricole; infatti, se da un lato gli aspetti funzionali sono particolarmente determinanti, tanto da avere spinto a descrivere questa architettura come "pura, astilistica e funzionale" ${ }^{10}$, dall'altro l'interpretazione funzionalista risulta troppo riduttiva, in quanto da sola non può comprendere taluni fattori come, ad esempio, il clima locale o la specifica reperibilità di materiali da costruzione.

L'architettura rurale non comprende soltanto gli edifici necessari alle abitazioni per gli uomini, i magazzini per la conservazione dei prodotti, i locali per la loro lavorazione, i ricoveri per gli animali da allevamento, $i$ depositi per gli attrezzi e le macchine, ma anche qualunque trasformazione fisica operata dall'uomo per potere svolgere le attività produttive in campagna. Pertanto, ogni edificio rurale si dovrebbe osservare come elemento significativo di un sistema più vasto, all'interno del quale trova motivo di esistere ogni sua componente, comprese, anche, le opere idrauliche, la viabilità aziendale, le sistemazioni orografiche

\footnotetext{
${ }^{9}$ L. 5 dicembre 1985 n. 730.
}

${ }^{10}$ Pagano/Daniel, 1936. 
destinate ad accogliere le colture o a consolidare il terreno, le chiusure dei campi, e così via. Ciò comporta che un'attenta lettura dell'architettura rurale dovrebbe comprendere anche il relativo paesaggio agrario cioè quell'impronta che, nel corso del tempo e ai fini delle sue attività produttive e agricole, l'uomo imprime sistematicamente al paesaggio naturale ${ }^{11}$. Tuttavia, l'assenza di una precisa identità per questo tipo di architettura non deve far pensare che non ci sia dietro la presenza di un'attività progettuale, la quale si manifesta nella sua tendenza alla regolarizzazione ed è interpretabile non solo come frutto di una migliore funzionalità delle forme regolari, ma anche come la conseguenza di esigenze estetiche. Nel corso del tempo la realizzazione di manuali e di trattati ha influito sull'evoluzione di un modulo basilare, in quanto, a partire dalla tipologia architettonica, dove si rifletterebbero soprattutto le funzioni economiche, fino ad arrivare alle componenti formali, dove si riconoscerebbero le influenze dell'ambiente cittadino, svariati condizionamenti gravano sul mondo contadino. L'architettura rurale ha tratto le sue radici da popoli diversi che sin dall'antichità hanno sentito la necessità di adeguare e migliorare il costruito, tenendo conto delle specifiche esigenze legate alle attività agricole ${ }^{12}$.

Per definire l'architettura rurale, é necessario fare alcune precisazioni sul termine "rurale". La definizione di "relativo alla campagna" non è sufficiente a descrivere l'architettura rurale, in quanto presuppone che esista una netta separazione tra città e campagna. Di fatto, non esistendo tale separazione, come anticipato parlando di territorio periurbano, non si può considerare elemento qualificante l'uno o l'altro termine. Infatti, l'agricoltura non ha mai posseduto l'esclusivo dominio del territorio extraurbano; basti pensare, sin dai tempi più remoti, agli insediamenti legati alle attività artigianali e industriali o alle attività ricreative, $\mathrm{o}$ ancora alle infrastrutture come strade, ponti e porti ${ }^{13}$.

Il predominio culturale dei modelli urbani, ha imposto il riferimento, come luogo comune, a categorie come la "spontaneità" o la "rusticità" che hanno evidenziato soltanto gli aspetti superficiali e non caratteristici dell'architettura rurale, come la rozzezza, l'irregolarità o il linguaggio vernacolare. Al contrario, è vero che l'architettura rurale comprende gli edifici costruiti in campagna, ma ad opera di tecnici colti ed in base a progetti ben organizzati, redatti al fine di sopperire ai bisogni dell'agricoltura e dell'azienda agricola, oltre che alla razionale abitazione dei contadini; una simile distinzione, anche se è stata avanzata in epoca recente, si può considerare valida anche per gli edifici del passato ${ }^{14}$.

\section{Caso studio}

\section{Premessa e metodologia di indagine}

In questa sezione vengono presentati i risultati dell'analisi di un caso studio per valutare l'influenza concreta nel territorio rurale siciliano degli aiuti comunitari, cioè l'apporto delle PC ad una pianificazione territoriale che consenta il ripristino e la valorizzazione dei fabbricati dell'architettura rurale, al fine di valorizzare efficacemente il paesaggio e di promuovere uno sviluppo organico del territorio.

Si presenta, come caso studio, il rilevante e peculiare progetto di ristrutturazione di un complesso rurale esistente nel territorio del Comune di Santa Ninfa in Provincia di Trapani, di proprietà della ditta Cirrincione Domenica, al fine di realizzare una struttura ricettiva agrituristica, fortemente caratterizzata dal calore, dall'essenzialità, dalla purezza di stile e dalla naturalità dei materiali impiegati, necessari al particolare orientamento al benessere psicofisico ricercato.

La metodologia di indagine utilizza il criterio della ricerca sul campo e di tipo documentale, al fine di deliniare le peculiarità pregresse del territorio, del fabbricato rurale e dell'azienda agricola; quindi viene effettuato il rilievo metrico e fotografico dell'esistente. Sono condotte un'analisi storica del territorio, ricerche cartografiche e acquisizione di mappe

\footnotetext{
11 Sereni, 1961: 29.

12 Crova, 1942.

${ }^{13} \mathrm{Ugo}, 1984$.

${ }^{14}$ Marconi, 1936.
} 
utili al fine di individuare il manufatto architettonico ed inquadrarlo nel territorio del paesaggio rurale locale ${ }^{15}$; quindi, anche mediante lo studio della documentazione progettuale è analizzato lo stato dei fabbricati ante e post intervento effettuato sfruttando le risorse fornie dal bando di finanziamento della Comunità Europea sopra menzionato.

Gli interventi edilizi, appresso esplicitati, sono finalizzati alla riqualificazione architettonica dei fabbricati, al loro più armonico adattamento all'ambiente ed alla tradizione architettonica locale, nel rispetto del genius loci tipico di un'area protetta, estesa oltre 17 ettari, di straordinario interesse geologico e naturalistico che mantiene la sua vocazione agricola compatibile con le esigenze di preservazione della biodiversità locale. Il tutto si inquadra all'interno della Riserva Naturale Integrale "Grotte di Santa Ninfa" e a ridosso di un territorio molto ampio di rimboschimento affidato alla gestione del Corpo Forestale dello Stato.

Gli interventi, che hanno portato alla realizzazione di un agriturismo, sono stati finanziati mediante il P.S.R. Sicilia 2007/2013 - Misura 311 azione A - Agriturismo, D.D.S. n ${ }^{\circ}$ $3071 / 2010$ del 22/12/2010.

\section{Procedimento amministrativo e titoli abilitativi}

In sede di Conferenza dei Servizi, indetta dall'Assessorato Regionale al Territorio ed Ambiente, ai sensi dell'art. 14 della Legge 07/08/1990 n. 241 e s.m.i. e dell'art. 122 della L.R. 07/05/2001 n. 6 e s.m.i., tenutasi il giorno 16/02/2007 presso i locali del Dipartimento Regionale Territorio ed Ambiente, è stato approvato il progetto di ristrutturazione edilizia e cambio di destinazione d'uso di un fabbricato rurale esistente da destinare a struttura ricettiva per turismo rurale. Per lo stesso progetto, è stata rilasciata dal Comune di Santa Ninfa la conseguente Concessione Edilizia n. 10/2007 del 20/03/2007.

Succesivamente la ditta proprietaria, intendendo variare la tipologia dell'attività ricettiva da turismo rurale ad agriturismo, ha richiesto all'Assessorato Territorio ed Ambiente il relativo Nulla Osta. Con nota n. 31087 del 06/05/2010, l'Assessorato Territorio ed Ambiente - Dipartimento Regionale per l'Ambiente - Servizio 6T - Protezione Patrimonio Naturale, ha espresso formale Nulla Osta sulla circostanza che la tipologia dell'attività ricettiva venga variata da turismo rurale ad agriturismo, mantenendo immutate le condizioni e le prescrizioni originarie.

Soggetto proponente e gestore della iniziativa è la ditta individuale Cirrincione Domenica, nata a Palermo il 18 febbraio 1978, C.F. CRRDNC78B58G273Z e residente in Milano nella Piazza dell'Assunta n. 1/A, 20141 Milano, in forza di regolare preliminare di compravendita notaio M.A Lo Piccolo in Palermo il 07/06/2004 con rep. N. 114421 (fig. 2).

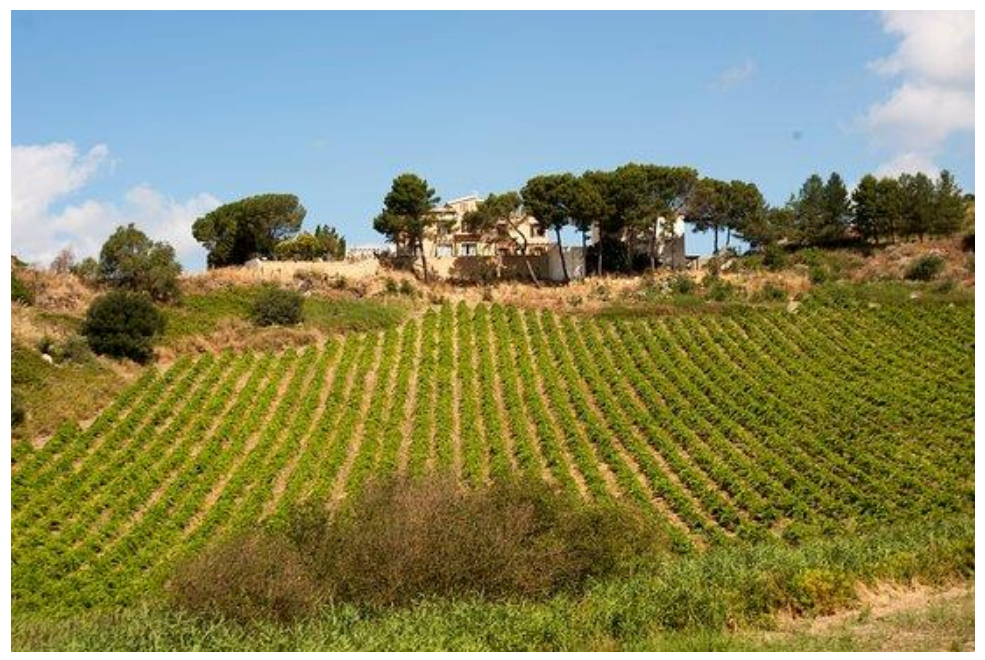

Fig. 2. Vista dell'inserimento nel paesaggio rurale

${ }^{15}$ Revenga, 2012: 125-126. 


\section{Inquadramento territoriale}

L'immobile da ristrutturare, identificato nelle cartografie I.G.M. con il toponimo di "Case Martino" sorge a Sud della Montagna della Magione, ai margini di un vasto fondo aziendale attualmente coltivato a vigneto e ricoperto naturalmente dalla tipica macchia mediterranea, è sito nel comune di Santa Ninfa (prov. di Trapani), in c.da Biviere Scarlatta, che si estende trasversalmente lungo tutto il vallone del Biviere. Attualmente l'intera proprietà fondiaria risulta annotata nel N.C.T. di Santa Ninfa al foglio di mappa n. 28, particelle nn. 40, 41, 42, 49, 56, 105, 106, 107, 111, 112, 113, 114, 115, 116, 117, 274, 275, 276, 277, 467 e 468; la superficie catastale complessiva è di 17 ettari, 31 are e 87 centiare (ha 17.31.87). La superficie territoriale $\left(\mathrm{S}_{\mathrm{t}}\right)$ del fondo è pertanto di $173.187 \mathrm{~m}^{2}$.

L'intera proprietà, secondo le previsioni del vigente P.R.G. del Comune di Santa Ninfa, approvato con Decreto Assessoriale 3 aprile 2000, ricade interamente in Zona Agricola "E1", le cui norme tecniche di attuazione prescrivono:

Densità Fondiaria $\left(\mathrm{D}_{\mathrm{f}}\right)=0,03 \mathrm{mc} / \mathrm{mq}$

Altezza massima $(\mathrm{H}) \quad=7,50 \mathrm{ml}$.

Numero piani fuori terra $\left(\mathrm{N}_{\mathrm{p}}\right) \quad=2$

Distanza dai confini $(\mathrm{D})=10,00 \mathrm{ml}$.

Gli immobili da ristrutturare ricadono inoltre:

- in prossimità del limite di confine della Riserva Naturale Integrale della Grotta di Santa Ninfa, istituita dall'Assessorato Regionale al Territorio e Ambiente con D.A. 16 maggio 1995 ai sensi dell'art. 4 della L.R. ${ }^{\circ} 14 / 88$.

- in prossimità del limite di confine del Sito di Importanza Comunitaria "Complesso dei Monti di Santa Ninfa e Gibellina e Grotta di Santa Ninfa", istituito nel 1998 nell'ambito del progetto BioItaly per la presenza di diversi habitat di interesse comunitario e di specie di interesse biogeografico e conservazionistico ai sensi della direttiva Habitat 92/43/CEE.

\section{Descrizione degli immobili nello stato ante intervento}

Posti ai margini della vasta proprietà aziendale, sul confine con la strada vicinale Biviere, (traversa lato Nord della Strada Statale n.119) sorgono tre fabbricati rurali di differente epoca di realizzazione, dei quali solo quello denominato Corpo A, sarà interessato ai lavori edili di ristrutturazione ed al cambio di destinazione d'uso. L'immobile infatti è stato costruito conformemente al regolare Nulla Osta per l'Esecuzione di Lavori Edili rilasciato a seguito di parere favorevole della commissione edilizia del 30/01/1970, e di Autorizzazione del Genio Civile n. 1256 del 11/04/1970. L'edificio si compone nel suo insieme di due distinte unità abitative:

-la prima, composta al piano terra da una cucina - pranzo, una camera letto, un bagno, ed al piano primo da due camere da letto e da un ripostiglio, collegati verticalmente da un primo vano scala;

-la seconda, composta al piano terra da un ampio vano destinato a magazzino-garage per il ricovero dei mezzi agricoli, dal quale si accede ad un piccolo vano e ad un bagno; al piano primo da un ingresso-soggiorno, un vano cucina, un bagno, tre camere da letto, un ripostiglio; al piano secondo sono ubicati un ulteriore vano ed una mansarda sottotetto; un secondo vano scala, collega verticalmente i tre livelli dell'edificio, afferenti alla seconda unità abitativa.

Il fabbricato, a tre elevazioni fuori terra, ha una struttura portante intelaiata in calcestruzzo armato a maglie chiuse. Le fondazioni, poste sopra uno strato di magrone sono composte da travi rovesce a $\mathrm{T}$, sempre in conglomerato cementizio armato. I solai di interpiano sono in latero cemento con travetti prefabbricati, laterizi forati di alleggerimento e caldana gettata in opera. I solai di copertura, anch'essi in latero-cemento sono in parte piani, a terrazza pavimentata in mattoni di cemento, ed in parte a doppia falda inclinata. I collegamenti verticali tra i vari piani sono garantiti da due scale in cemento armato a soletta piena. Le quote di interpiano sono di 4,00 m. per il piano terra, di 3,20 m. per il piano primo, e di m. 2,80 per il secondo. La muratura di tamponamento è realizzata in conci di tufo legati con malta bastarda, mentre la tramezzatura interna è in segati di tufo. Tutti gli ambienti interni delle 
due unità abitative presentano pareti e soffitti intonacati al civile, ad esclusione delle pareti del deposito di piano terra che sono semplicemente rinzaffate. Il prospetto esterno è intonacato con ultima mano di decorativo del tipo "plastico". Gli infissi interni realizzati con anta e telaio in legno, mentre gli infissi esterni in alluminio pre-verniciato sono dotati di persiana. I due portoni di accesso sono in legno. Le unità abitative presentano inoltre pavimenti in ceramica o in marmette pressate di cemento con scaglie di marmo, e sono dotati di impianto elettrico e di riscaldamento.

Il corpo A occupa una superficie coperta di 209,40 $\mathrm{m}^{2}$ al piano terra, di 209,40 $\mathrm{m}^{2}$ al piano primo, e di $128,42 \mathrm{~m}^{2}$ (di cui $94,50 \mathrm{~m}^{2}$ quale area sottotetto stenditoio) al piano secondo, mentre il volume è di $1668,08 \mathrm{~m}^{3}$. L'altezza alla massima linea di gronda attuale è di 9,70 $\mathrm{m}$, mentre quella alla massima linea di colmo è di 11,00 m.

\section{Descrizione degli immobili post intervento}

L'ingresso alla struttura ricettiva avviene attraverso la terrazza belvedere, quindi si attraversa la hall del corpo A (fig. 3). Dalla sala d'ingresso per gli ospiti, articolata in zone riservate alla lettura ed alla conversazione, si accede mediante una scala in c.a. alle camere poste al piano terra, primo e secondo. Si può accedere inoltre ad una terrazza panoramica interna, che avvolge i fabbricati esistenti e prospetta sulla splendida valle.

Gli interventi edilizi necessari per adeguare gli i mmobili alla nuova destinazione d'uso hanno previsto la realizzazione nel corpo A delle camere per gli ospiti e di alcuni locali di utilizzo comune. La nuova distribuzione planimetrica e volumetrica prevede che al piano terra del corpo A, in corrispondenza degli ex magazzini, siano presenti i locali della sala ristorante e prima colazione con annessa cucina, ed i servizi per il personale e per gli ospiti. Complessivamente sono presenti n. 21 posti. Le dimensioni della sala ristorante, al netto della cucina e dei servizi per il personale, soddisfano i requisiti minimi dimensionali fissati dal punto 8.3 comma 5 del Decreto 10/08/2009 dell'A ssessorato Agricoltura e Foreste.

Il salone è aperto, attraverso ampie vetrate, direttamente sulla terrazza panoramica. Un pergolato, realizzato con struttura in legno costituita da pilastri verticali di sezione $15 \times 15 \mathrm{~cm}$ e travi orizzontali di sezione $18 \times 10 \mathrm{~cm}$ e listelli di sezione $5 \times 5 \mathrm{~cm}$, ricoperto con copertura piante rampicanti (Vitis vinifera e Clematis cirrhosa) che funge da filtro tra gli ambienti interni e quelli esterni. Lateralmente inoltre, è presente una zona degustazione coperta anch'essa da con un pergolato realizzato con le medesime modalità del precedente (fig. 4).

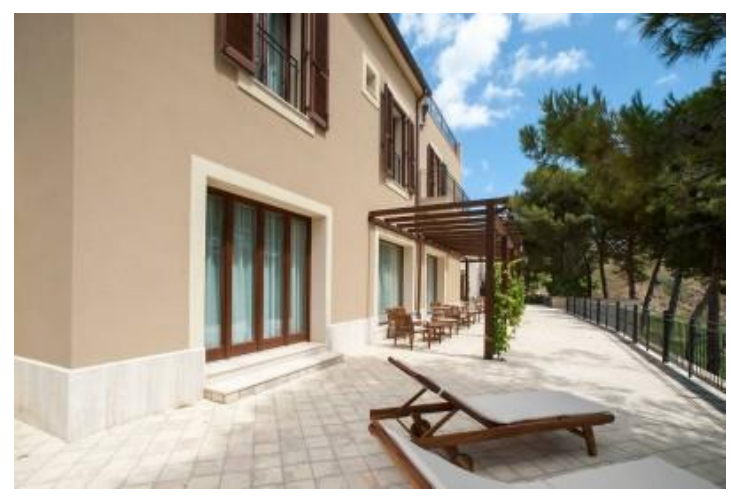

Fig. 3. Vista della facciata interna, Rubino Domenico

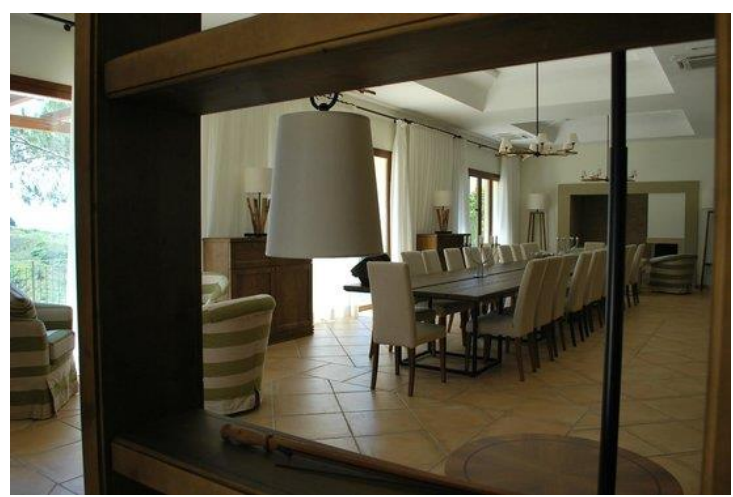

Fig. 4. Vista del salone, Cirrincione Domenica

Al piano terra del corpo A sono presenti le prime due camere doppie per ospiti, entrambe dotate di bagno privato interno, una delle quali avente il requisito di accessibilità da parte di persone con ridotta o impedita capacità motoria e sensoriale. Al piano primo, al posto delle preesistenti unità abitative, sono state realizzate n. 6 camere doppie, n. 1 camera tripla, tutte provviste di bagno privato interno, di cui uno con aerazione forzata ed i restanti con 
aerazione naturale diretta dall'esterno, un locale deposito biancheria di piano (fig. 5). Al secondo piano è presente una camera doppia, provvista di bagno privato interno, e due terrazze panoramiche (fig. 6).

Complessivamente la nuova struttura ricettiva dispone di 10 camere di cui 9 doppie e 1 tripla per un totale di 21 posti letto.

Nella progettazione dei vari ambienti si è anche tenuto conto:

- delle "Regole tecniche di prevenzione incendi per le strutture alberghiere" Decreto n. 9/04/1994 e successive modifiche ed integrazioni, emanato dal Ministero dell'Interno.

- del D.M.236/89 e L.N.13/89 in materia di accessibilità da parte di persone con ridotta o impedita capacità motoria o sensoriale.

Ad intervento realizzato la superficie utile interna ed il volume delle strutture immobiliari oggetto dell'intervento saranno immutate rispetto a quelle dello stato di fatto.

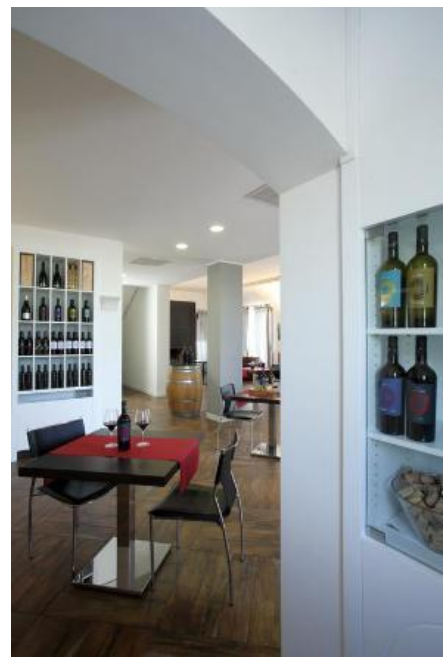

Fig. 5. Vista della sala consumazione, Cirrincione Domenica

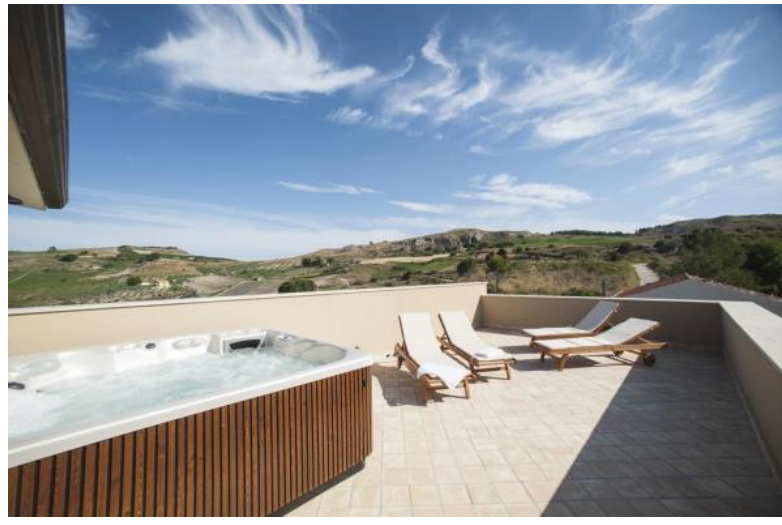

Fig. 6. Vista della terrazza di secondo piano, Cirrincione Domenica

Aspetti progettuali dell'intervento

La filosofia che inspira l'intervento progettuale nasce dalla presenza intorno ai fabbricati in oggetto di un ambito naturale e paesaggistico di primaria importanza e qualità. Ci si prefigge, quindi, l'obiettivo di realizzare una struttura ricettiva alberghiera che per forma, carattere, colori, riesca a produrre un organismo architettonico perfettamente integrato nell'ambito naturale ove è collocato, e che garantisca il mantenimento, il rispetto e la tutela delle tipologie e delle forme dei manufatti tradizionali, in un contesto di valorizzazione delle risorse locali al fine di preservare il paesaggio locale (fig. 7).

Per raggiungere il soprindicato obiettivo sono state seguite precise regole e scelte progettuali:

A) L’utilizzo di materiali e di tecniche che riducano la quantità di energia utilizzata dalla struttura, e mirati ad un risparmio energetico e idrico mediante l'approvvigionamento da fonti energetiche alternative, il riutilizzo di acque meteoriche ed alla gestione razionale e differenziata dei rifiuti.

A tal proposito è presente:

- un sistema di produzione di acqua calda sanitaria che, mediante n. 12 pannelli solari, è in grado di ottenere dal sole almeno il $70 \%$ dell'energia complessiva utilizzata per la produzione di acqua calda; 
- la riduzione di consumo di acqua per lo scarico di tutti i WC presenti mediante l'impiego di cassette a zaino a due scomparti da 6 litri e 4 litri comandati in alternativa da doppio pulsante;

- un circuito differenziato per l'alimentazione idrica delle cassette a zaino per lo scarico dei wc, che utilizza quale sorgente l'acqua proveniente da una cisterna ove vengono raccolte e decantate le acque meteroriche provenienti dalle falde e dalle terrazze di copertura;

- un sistema di raccolta differenziata dei rifiuti, all'interno della struttura ricettiva, che permetta di separare carta, plastica, vetro, latta e lattine ed organico in appositi contenitori, forniti gratuitamente dall'ente gestore, e stoccati temporaneamente in una isola ecologica protetta, posta in area limitrofa all'azienda;

- un pozzetto degrassatore dove le miscele di acqua con sostanze oleose non in emulsione e solidi galleggianti, provenienti dalle cucine, si separano per gravità in modo continuo e naturale.

B) Sviluppo sostenibile dell'intervento di ristrutturazione mirato all'ottenimento delle certificazioni di qualità ambientale tipo "ECOLABEL", e all'impiego di tecniche rispettose dell'ambiente e di bioarchitettura, a basso impatto ambientale e paesaggistico, utilizzando materiali naturali, sostituendo i rivestimenti esistenti con nuovi materiali salutari per l'uomo ed eseguendo opere edili per il preciso intervento di migliorare l'inserimento dell'immobile nel paesaggio naturale.

A tal proposito si è previsto di:

- realizzare una struttura alberghiera con un minore impatto ambientale rinunciando ad ogni forma di ampliamento plano-volumetrico;

- impiegare per la tinteggiatura delle pareti idropittura lavabile a struttura microcristallina a base di silicati di potassio, anallergica, formulata con materie prime naturali, tipo acqua, carbonato di calcio idonea per interventi bioedili e nel restauro di edifici d'epoca;

- in un'ottica bioecologica dell'intervento, sostituire l'intonaco plastico del prospetto esterno con uno strato di intonaco del tipo cocciopesto costituito da cotto siciliano macinato, sabbie laviche, e calce idraulica, idoneo per restauri e recuperi architettonici interni ed esterni;

- sostituire nella copertura a falde esistente, il sovrastato di tegole marsigliese in laterizio di colore rosso che mal si integra nel territorio e rappresenta un impatto ambientale negativo nel tessuto agreste, con una copertura in coppi siciliani di argilla tipici della cultura architettonica del luogo ed in grado di migliorare l'inserimento nel paesaggio naturale dell'immobile. Tale intervento edilizio è stato oltretutto espressamente richiesto dalla competente Soprintendenza ai Beni Culturali e Ambientali di Trapani;

- sostituire gli infissi in alluminio di colore bianco esistenti con nuovi infissi in legno massello, completi di persiane esterne e anta a vetro camera interna, protette con vernici all'acqua.

Gli interventi di ristrutturazione hanno previsto una serie di demolizioni parziali o totali di tratti di muratura esistente per consentire l'apertura di nuovi vani porta, l'allineamento e l'ampliamento di vani porta o finestra. Si è inoltre proceduto alla demolizione dei tramezzi esistenti e alla realizzazione di una nuova tramezzatura interna con tavelle in calcestruzzo leggero di argilla e pomice. È stata demolita una delle due scale in c.a. per consentire la realizzazione di un nuovo solaio e quindi di una camera per ospiti. Il nuovo solaio, ancorato alle travi perimetrali della struttura è stato realizzato in latero cemento con caldana in opera.

L'approvvigionamento idrico è garantito dalla presenza all'interno dell'area libera di una vasca di riserva idrica della capacità complessiva di 20.000 litri il cui rifornimento è garantito da fornitura comunale convenzionata tramite autobotte. Le acque prelevate da una pompa sommersa, tramite un'autoclave centralizzata dimensionata per garantire le pressioni minime di legge su tutti i punti di erogazione, sono distribuite a tutte le utenze della struttura ricettiva. La vasca presenta pareti rivestite con vernici epossidriche atossiche per alimenti.

Non essendo la zona fornita di rete fognaria comunale, il sistema di smaltimento dei reflui, conformemente a quanto previsto dalla vigente normativa in presenza di insediamenti 
inferiori a 50 abitanti equivalenti, è stato realizzato un sistema di depurazione primaria tramite vasca tipo Imhoff, e successivo stoccaggio in vasca a tenuta.

L'illuminazione di tutti i locali è del tipo naturale e artificiale: l'illuminazione naturale è garantita dalle superficie finestrate ad anta apribile, aventi dimensioni superiori ai parametri previsti dai vigenti articoli del locale Regolamento Edilizio; l'illuminazione artificiale interna è diretta, garantita da apparecchi illuminanti posti a soffitto, costituita da lampade fluorescenti o a Led a basso consumo. In tutti i locali sono installati apparecchi fluorescenti per l'illuminazione di emergenza.

L'impianto di riscaldamento-condizionamento è del tipo a pompa di calore, a funzionamento elettrico, composto da centralina di distribuzione, unità esterna e unità interna a ventilconvettori regolabili manualmente dall'interno.

Aspetti multifunzionali dell'intervento e piano turistico

Il progetto ha previsto la realizzazione di un'oasi di vacanza defaticante basata sul principio del riavvicinamento all'ambiente naturale e a se stessi ed in grado di consentire a chi vi soggiorna un rapido recupero del rapporto con la natura, con il proprio corpo e con la propria mente.

Si tratta, nello specifico, di una struttura di circa 21 posti letto di livello medio-alto (assimilabile sul piano normativo ad un 3 stelle) con ristorazione riservata agli ospiti, dotata di servizi per il tempo libero ed il benessere (biblioteca/sala lettura, ecc.) e di supporto (area ristorazione), rivolto ad una clientela internazionale con residenza prevalente nei grandi centri urbani; un resort di campagna realizzato e gestito in accordo con gli "Orientamenti di base per il turismo sostenibile in Europa" della Commissione Europea. Rivolto ad una clientela internazionale (europea) ad alto livello di scolarizzazione con residenza prevalente nei grandi centri urbani e capacità di acquisto media; una costruzione realizzata attraverso la ristrutturazione di fabbricati rurali esistenti migliorati per adattarli al contesto paesaggistico, per migliorarne l'inserimento e la compatibilità ambientale, le caratteristiche e l'aspetto estetico (fig. 8).

Il complesso ricettivo si pone a disposizione del territorio aperto, pur nel rispetto del suo orientamento strategico al benessere psicofisico, alle esigenze ricettive legate allo studio ed alla gestione della "Riserva Naturale Integrale della Grotta di Santa Ninfa" da parte dell'Ente Gestore Legambiente, oltre che alla vivace presenza artistica nel territorio che ha il suo fulcro nell'attività della Fondazione Orestiadi.

La struttura ha conseguito notevoli successi economici nel campo di ricettività turistica; infatti, la titolare dell'azienda agricola svolge come attività principale quella di operatore del turismo ed è esperta di promozione, commercializzazione e distribuzione di strutture ricettive e destinazioni, attività svolta sia per conto di operatori italiani che internazionali. L'attività è riuscita a posizionarsi correttamente e a sostenere le assumption relative a tariffe $\mathrm{e}$ occupazione posti letto, anche grazie al significativo investimento effettuato sul fronte della tecnologia sia gestionale (hardware e software) sia distributiva (accesso a sistemi di prenotazione e distribuzione). Ne sono riprova l'adesione già contrattualizzata ad una catena alberghiera che raggruppa, sotto vari brand, strutture agrituristiche e strutture alberghiere rivolta alla clientela in cerca di esperienze di soggiorno che riconducano al benessere psicofisico ed al riavvicinamento all'ambiente naturale.

\section{Considerazioni finali}

Il contributo, mediante l'analisi del territorio, l'esame degli elaborati progettuali ed il rilievo dei manufatti rurali, lo studio bibliografico e della normativa comunitaria, ha consentito di delineare le peculiarità dei luoghi indagati, ponendo l'accento sul contributo fornito dalle politiche comunitarie all'evoluzione dello sviluppo urbanistico e architettonico del territorio, anche dal punto di vista del nuovo posizionamento in un'ottica multifunzionale delle attività legate all'agricoltura e al territorio rurale stesso.

Come si è osservato, sulla nuova concezione dei beni culturali si è innestata una nuova visione dell'intervento pubblico in materia, consistente nel passaggio da un'attività di tutela 
statica del bene ad un intervento diretto a garantire alla collettività una fruizione ampia ed effettiva del valore e del significato culturale custodito. È chiaro quanto risulti fondamentale che l'intervento pubblico sui beni culturali attribuisca sempre più rilievo alle attività dirette a favorire la fruizione collettiva dei beni culturali, e cioè alle attività di valorizzazione e di gestione, e non di sola tutela. L'esigenza di tale tutela trova una sua ragione d'essere nella natura stessa dell'Italia e della Sicilia in particolare, dal suo essere espressione di un patrimonio storico, artistico e culturale senza eguali.

Lo sviluppo della ricerca evidenzia l'interesse e l'importanza che possono rivestire le politiche europee nel recupero del paesaggio rurale e delle diverse tipologie costruttive del patrimonio edilizio rurale, mediante interventi di restauro e ristrutturazione dell'esistente che mantengano la destinazione d'uso originaria, o di riqualifica dello stesso patrimonio ad un utilizzo ad essa compatibile, quale l'agriturismo, come nel caso in questione, l'opificio rurale, la fattoria didattica o altro, consentendo uno sviluppo integrato e sostenibile, e puntando ad approccio che tenda al progresso della multifunzionalità in agricoltura.

Infine, l'esame dettagliato di alcuni interventi già mostra elementi avvaloranti l'ipotesi dell'influenza positiva delle PC allo sviluppo del territorio rurale. Non è ancora chiaro se tale influenza sia di carattere generale, ossia contribuisca allo sviluppo integrato del territorio, o piuttosto contenuta e distribuita non uniformemente nel territorio, ossia indirizzata a investimenti che promuovono lo sviluppo di singole aziende e che non riescono a fare sistema al fine di uno sviluppo integrato del territorio. Per dare risposta a tale quesito, si attendono nuovi esiti, anche relativi alla pianificazione legata al PSR 2014/2020 ancora in fase di sviluppo iniziale. 


\section{Bibliografía}

Antrop, M.: "Changing patterns in the urbanized countryside of western Europe". En: Landscape Ecology, [Núm.]15, (2000), pp. 257-270.

Antrop, M.: "Landscape change and the urbanisation process in Europe". En: Landscape and Urban Planning, [Núm.]67, (2004), pp. 9-26.

Antrop, M./Van Eetvelde, V.: "Holistic aspect of suburban landscape: visual image interpretation and landscape metrics”. En: Landscape and Urban Planning, [Núm.]50, (2000), pp. 43-58.

Berger, A./Chevalier, P./Cortes, G./Dedeire, M. (eds.): Patrimoine héritages et développement rural en Europe. Paris: L'Harmattan, (2010), pp. 7-16.

Catone, Marco Porcio: "De agricoltura"/Varrone, Marco Terenzio (37 a.C.): "De re rustica"/Vitruvio, Marco (15 a.C:) "De architectura". En: Crova, B: Edilizia e tecnica rurale di Roma antica. Milano: Bocca Ed. (1942).

Cedrini, R.:"Per una lettura antropologica del baglio". En: Pilato, G/Tinorio, P./Gargagliano, L. (eds.): Progetto di sviluppo economico dei territorio di Paceco. Recupero delle preesistenze storico-orientali. Palermo, (1997), p. 13.

Ferroni, F.: "Biodiversità e governo del territorio nelle strategie e politiche internazionali". En: Ferroni, F./Romano, B. (eds.): Biodiversità, consumo di suolo e reti ecologiche. La conservazione della natura nel governo del territorio. WWF Italia, Ministero dell'Università e della Ricerca Scientifica: Cogecstre, (2010), pp.35-51.

Marconi, P.: "Architettura rustica" (s.v.). En: AA. VV. Enciclopedia Italiana, vol. XXX. Roma:Treccani. (1936).

OECD : OECD Regional Typology. Paris: OECD Publishing. (2011)

OECD . Creating Rural Indicator for shaping territorial policy. Paris: OECD Publishing. (1994) ONU : 2011 Demographic Yearbook. New York: ONU. (2012)

ONU : Glossary of Environment Statistics. New York: United Nations Publication. (1997)

Pagano, G./Daniel, G.:"Architettura rurale in Italia”. En Quaderni della Triennale. Milano:Hoepli editore. (1936).

Revenga Domínguez, P.: "Hacia una adecuada utilización del Patrimonio como recurso turístico", Areté Documenta. Boletín de la Asociación Española de Gestores del Patrimonio, 16, (2002), pp. 113-124.

Revenga Domínguez, P.: "Metodologías, interpretaciones y tributos de la Historia del Arte", en Palacio Prieto, J.L. (coord.): 90 años de cultura, México, UNAM, 2012, (2012), pp. 87126.

Santini, C.: "Costruzioni rurali" (s.v.). En: AA. VV.: Enciclopedia Italiana, vol. XXX. Roma: Treccani. (1936).

Sereni, E. Storia del paesaggio agrario italiano. Bari: Lateza, (1936), p. 29

Slemp, C. et alt.: "Growing too fast: Local stakeholders speak out about growth and its consequences for community weel-being in the urban-rural interface". En: Landscape and Urban Planning, [Vol.] 106, (2012), pp. 139-148.

Ugo, V.: Paesaggio, architettura. Palermo: Cogras. .(1984).

Zeng, H./Sui, D./Li, S.: "Linking urban field theory with GIS and Remote Sensing to detect signature of rapid urbanization on the landscape: toward a new approach for characterizing urban sprawl”. En: Urban Geography, [Vol.] 26, (2005), pp. 410-434. 